\title{
'||||||||||||||||||||||||||||||||||||||||||||||||||||||||||||||||||||.
}

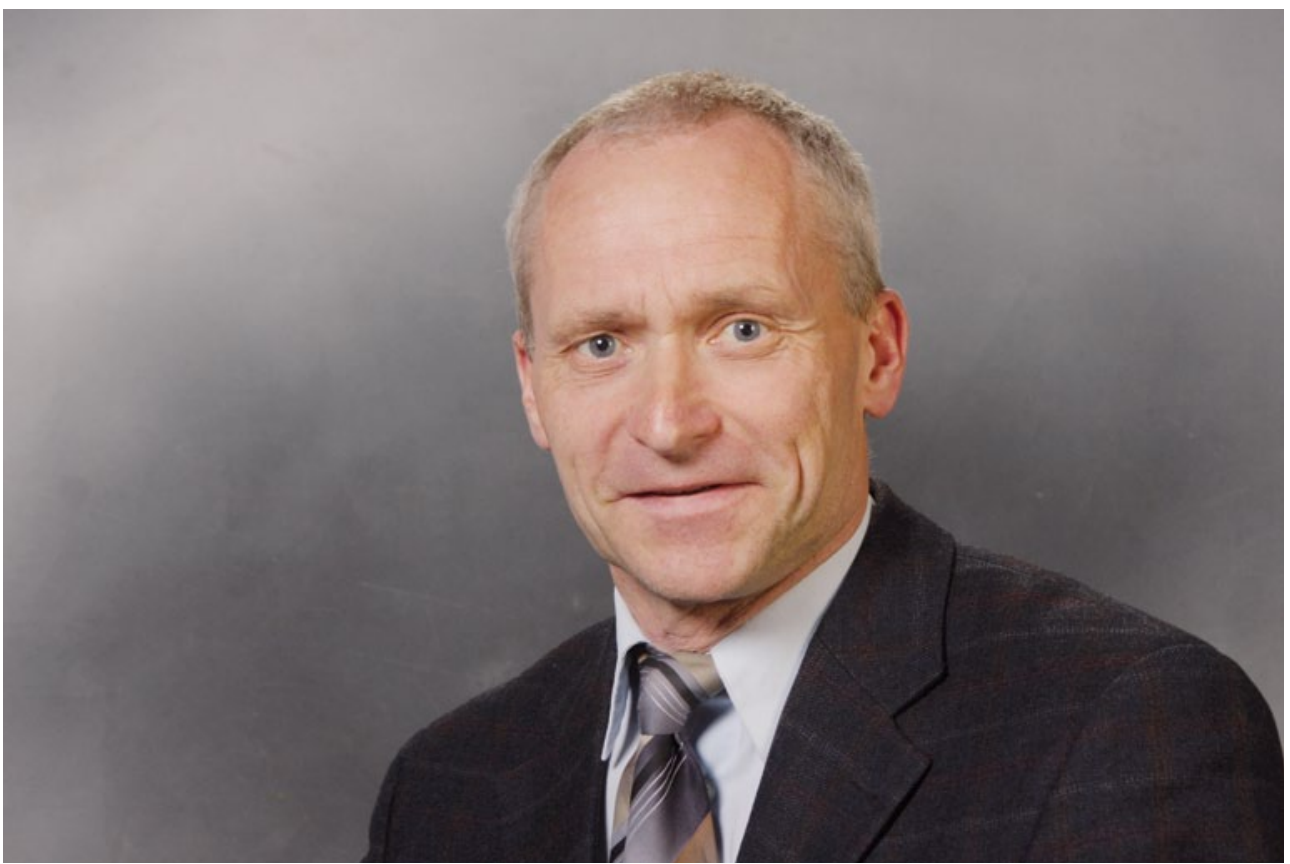

DR.-ING. UWE MEINIG

Leiter Entwicklung neue

Motor- und Getriebekomponenten,

SHW Automotive

\section{INNOVATIONEN UND SCHUTZRECHTE}

Die historische Würdigung namhafter Erfindungen, wie die von Nicolaus Otto, Gottlieb Daimler, Carl Benz, Rudolf Diesel oder Felix Wankel, rückt immer wieder auch die für die Erfinder nicht immer konfliktfreie, aber wichtige, überwiegend fachkundige Begleitung dieser Innovationen durch die Patentämter ins Bewusstsein. Auch wenn die heutigen Erfindungen überwiegend einen kleinteiligen Charakter besitzen, so kommt der Absicherung von innovativen Lösungen in der Produktentwicklung durch Patente und Gebrauchsmuster nach wie vor eine ganz wesentliche Bedeutung zu. Vor diesem Hintergrund muss es alarmieren, wenn ein kürzlich erschienener Artikel in den VDI-Nachrichten den Titel „Jedes zweite Patent in Deutschland ist potenziell rechtswidrig“ trägt. Darin wird die deutsche Patentrechtspraxis von Fachleuten sehr kritisch bewertet. Auch wenn man diesen Titel als überzogen einstuft, so gibt es doch in der praktischen Handhabung des Patentrechts berechtigte Kritikpunkte. Hierzu zählen die uneinheitlichen Maßstäbe, mit denen die erfinderische Tätigkeit beurteilt wird, oftmals in Verbindung mit vom Anmelder schwammig und vieldeutig formulierten Patentansprüchen, in vielen Fällen mit einer Kombination bunt zusammengestellter Merkmale, die nicht unmittelbar in einem Funktionszusammenhang stehen. Werden auf derartige Anmeldungen zu Unrecht Patente erteilt, obliegt es dem Wettbewerber, mit Mühen und Kosten gegen die in vielen Fällen objektiv nicht gerechtfertigte Erteilung vorzugehen. Dabei erweist sich bei Patentstreitigkeiten die Trennung von Verletzungsverfahren und Nichtigkeitsverfahren als eine besondere Problematik. Wird beispielsweise durch ein Produkt eines Unternehmens das Schutzrecht eines Wettbewerbers verletzt, so kann dieser Wettbewerber im Rahmen einer Unterlassungsklage verhältnismäßig kurzfristig durchsetzen, dass das Unternehmen das betreffende Produkt vom Markt nimmt, vorhandene Produkte vernichtet und die zugehörigen Marktdaten offenlegt, auch wenn sich möglicherweise Jahre später als Ergebnis einer Nichtigkeitsklage, gegebenenfalls auch gegen eine Zahl von Teilungsanmeldungen des ursprünglichen Patents, die nicht gerechtfertigte Erteilung des Schutzrechts herausstellt. Vor diesem Hintergrund ist es gefordert, dass sich die Patentämter in ihrer Erteilungspraxis den, aus einer Erteilung resultierenden, unter Umständen erheblichen rechtlichen und wirtschaftlichen Konsequenzen bewusst sind. Eine unzureichende Prüfung darf nicht mit Hinweis auf mögliche Rechtsmittel gegen die Erteilung abgetan werden.

Die aktuelle rechtliche Situation ermuntert einzelne, insbesondere größere Industrieunternehmen zu einer Patentstrategie, bei der von einer „Diensterfinderstruktur“ in großer Zahl Schutzrechte mit fragwürdiger Erfindungshöhe planmäßig ersonnen und vom Unternehmen angemeldet werden. Eine hohe Zahl von Anmeldungen kann schwerlich als Indikator für das Innovationspotenzial solch eines Unternehmens dienen.

Im Sinne der von der Politik immer wieder erhobenen Forderung nach Innovation steht hier auch der Gesetzgeber in der Pflicht. Ungerechtfertigte Monopolrechte stehen Innovationen entgegen. Gefordert ist ein modernes Patentrecht, das auf die Förderung und den Schutz wirklicher Innovationen zielt und nicht nur die Behinderung des Wettbewerbs bewirkt. 